\title{
Mitochondria-related reversal of early-stage diabetic nephropathy in donor kidney after transplantation in mice
}

\author{
Weinan $W_{u^{1,2,3,4,5 \#} \text {, Yiming Jin }{ }^{1,2,3,4,5,6 \#} \text {, Lisha Teng }}{ }^{1,2,3,4,5}$, Xue Shao ${ }^{1,2,3,4,5}$, Yucheng Wang ${ }^{1,2,3,4,5}$, \\ Shi Feng ${ }^{1,2,3,4,5}$, Cuili Wang ${ }^{1,2,3,4,5}$, Hong Jiang ${ }^{1,2,3,4,5}$, Jianyong $W_{u^{1,2,3,4,5}}$
}

${ }^{1}$ Kidney Disease Center, the First Affiliated Hospital, College of Medicine, Zhejiang University, Hangzhou 310003, China; ${ }^{2}$ Key Laboratory of Nephropathy, Zhejiang Province, Hangzhou 310003, China; ${ }^{3}$ Kidney Disease Immunology Laboratory, the Third-Grade Laboratory, State Administration of Traditional Chinese Medicine of China, Hangzhou 310000, China; ${ }^{4}$ Key Laboratory of Multiple Organ Transplantation, Ministry of Health of China, Hangzhou 310000, China; Institute of Nephropathy, Zhejiang University, Hangzhou 310003, China; ${ }^{6}$ Department of Nephropathy, Shaoxing Second Hospital, Shaoxing 312000, China

Contributions: (I) Conception and design: W Wu, Y Jin, H Jiang, J Wu; (II) Administrative support: J Wu; (III) Provision of study materials or patients: J Wu; (IV) Collection and assembly of data: W Wu, Y Jin; (V) Data analysis and interpretation: W Wu, L Teng, X Shao, Y Wang, S Feng, C Wang; (VI) Manuscript writing: All authors; (VII) Final approval of manuscript: All authors.

\#These authors contributed equally to this work.

Correspondence to: Prof. Jianyong Wu; Prof. Hong Jiang. Kidney Disease Center, the First Affiliated Hospital, College of Medicine, Zhejiang University, Qingchun Road 79, Hangzhou 310003, China. Email: wujianyong1964@zju.edu.cn; jianghong961106@zju.edu.cn.

Background: Renal diabetic changes are frequent in kidney transplantation (KTx) donors. Whether these diabetic changes are reversible remains a topic of debate. This study aimed to test the hypothesized reversibility of diabetic changes after KTx.

Methods: C57BL/6J mice were randomly divided into three groups: the control group, early-stage group (ESG), and advanced-stage group (ASG). Diabetes mellitus (DM) was induced in mice by intraperitoneal injection of streptozotocin (STZ) at $50 \mathrm{mg} / \mathrm{kg}$ body weight for five consecutive days. Blood glucose levels $\geq 16.7 \mathrm{mmol} / \mathrm{L}$ were indicative of diabetic mice. The kidneys from ESG and ASG were transplanted to control mice 12 or 32 weeks after STZ injection. Kidney tissue, blood, and 24-hour urine specimens of donor and recipient mice were collected before KTx and 28 days after KTx, respectively. We measured the body weight, blood glucose, histological changes, reactive oxygen species (ROS), apoptosis. Electron microscopy was also performed to evaluate the mitochondrial morphology. The expression of NADPH oxidases (NOXs) was assessed by qRT-PCR.

Results: Kidneys from early-stage diabetic mice showed evidence of lesion reversal four weeks after KTx, including decreased urinary albumin and reversal of histological changes. Besides, mitochondrial swelling, oxidative stress, apoptosis, and overexpression of NOXs in the kidneys were also suppressed. Conversely, no changes were observed in kidneys from advanced-stage diabetic mice after KTx.

Conclusions: We confirmed that early-stage but not advanced-stage diabetic nephropathy (DN) is reversible, which is related to reduced NOX expression and improvement in mitochondrial function. These results indicated that kidneys with early-stage DN could be used for KTx in clinical practice, as the disease may be reversed following KTx.

Keywords: Mitochondria; reversal; diabetic nephropathy (DN); diabetic donors; kidney transplantation (KTx)

Submitted Jul 26, 2019. Accepted for publication Nov 29, 2019.

doi: $10.21037 /$ atm.2019.12.55

View this article at: http://dx.doi.org/10.21037/atm.2019.12.55 


\section{Introduction}

Because of the increasing incidence of diabetes mellitus (DM), it is not uncommon for kidney donors to have DM at different stages (1). Particularly in cases involving cardiac death, the kidneys are often transplanted without complete clinical information regarding the donor. In kidneys from these donors, diabetic nephropathy $(\mathrm{DN})$ is usually not discovered until days after kidney transplantation (KTx) through pathological examination. A few studies have reported the reversibility of DN after KTx into recipients with normal blood glucose, but the reversibility remains under debate. Several researchers have discovered that strict control of glycemia could slow the worsening of renal function and reduce microalbuminuria in the early stages of DM (2-4). Fioretto and Mauer have reported that diabetic lesions in the kidneys of type $1 \mathrm{DM}$ patients are reversible after pancreatic transplantation alone $(5,6)$.

However, in the setting of KTx, there have been only two reports of DN reversal. In 1983, Abouna et al. reported a KTx case in which they transplanted a graft from a cadaveric donor with a 17 -year history of type 1 DM. They observed that the diabetic features, including diffuse glomerulosclerosis and thickening of the mesangial matrix and capillary basement membranes, had reversed almost completely 7 months after $\mathrm{KTx}$, as confirmed by renal biopsy (7). In 2015, Harada et al. reported that early diabetic changes in renal grafts from three donors with type 2 DM were reversed after KTx only when the recipients did not have diabetes and under reasonable glycemic control (8). As for animal studies, Lee et al. have reported that kidney isotransplantation from a rat with DM induced for 6 months into a healthy recipient resulted in inhibition or reversal of the glomerular lesions within 2 months (9).

The kidney is considered to have the second largest number of mitochondria per tissue mass among organs (10), and mitochondrial dysfunction has been demonstrated to play an essential role in DN (11). Hyperglycemia leads to an imbalance of NADPH production and consumption (12), resulting in excessive formation of reactive oxygen species (ROS) and mitochondrial injury. NADPH oxidases (NOXs) are transmembrane proteins that transport electrons across biological membranes to reduce oxygen to superoxide (13). The NOX family is overexpressed in DN (14) and related to ROS production (15). Damaged mitochondria can activate apoptosis through the extrinsic and intrinsic pathways. Susztak et al. reported that inhibition of NOXs attenuated ROS formation and prevented cell apoptosis (16).
In the present study, we investigated the hypothesis that only early-stage DN and not advanced-stage DN is reversible and that this phenomenon is related to mitochondrial function.

\section{Methods}

\section{Diabetic and transplantation animal model}

Eight-week-old male C57BL/6J mice (18-22 g body weight) were purchased from Zhejiang University Animal Center (Hangzhou, China) and housed under controlled environmental conditions (temperature $22{ }^{\circ} \mathrm{C}, 12$-hour light-dark cycle). Animals were provided free access to water and a standard laboratory diet. The animal experiments were approved by the Institution Ethics Committee for Investigation with Animals (Zhejiang University).

Mice were randomly divided into three groups: the control, early-stage (ESG), and advanced-stage group (ASG). DM was induced in the mice by intraperitoneal injection of streptozotocin (STZ, Sigma-Aldrich, Steinheim, Germany) at $50 \mathrm{mg} / \mathrm{kg}$ body weight in $0.1 \mathrm{mmol} / \mathrm{L}$ citrate buffer ( $\mathrm{pH} 4.5$ ) for five consecutive days. Bodyweight and blood glucose from tail vein collection were examined 3 days after the final injection and twice a week thereafter. Blood glucose levels $\geq 16.7 \mathrm{mmol} / \mathrm{L}$ were considered to indicate diabetic mice.

The C57BL/6J mice showed mild DN (mild basement membrane thickening and mesangial expansion) 10 weeks after STZ injection and advanced diabetic lesions (a mass of mesangial expansion, mesangial sclerosis, and bodyweight decline) after 30 weeks (17). The kidneys from ESG and ASG were transplanted to control mice 12 or 32 weeks after STZ injection, respectively, using previously described microsurgical techniques (18). Recipient mice were unilaterally transplanted. Mice were placed in metabolic cages for collection of 24-hour urine specimens 1 day before KTx and 28 days after KTx. Serum creatinine (Cre) and blood urea nitrogen (BUN) levels were determined by standard methods using an autoanalyzer (Hitachi 7600, Tokyo, Japan). Twenty-eight days after $\mathrm{KTx}$, mice were sacrificed, and renal tissue samples were processed and stored as needed. The animal experiment workflow is shown in Figure 1.

\section{Histological staining and measurement}

Renal samples collected at sacrifice were fixed by immersion in 


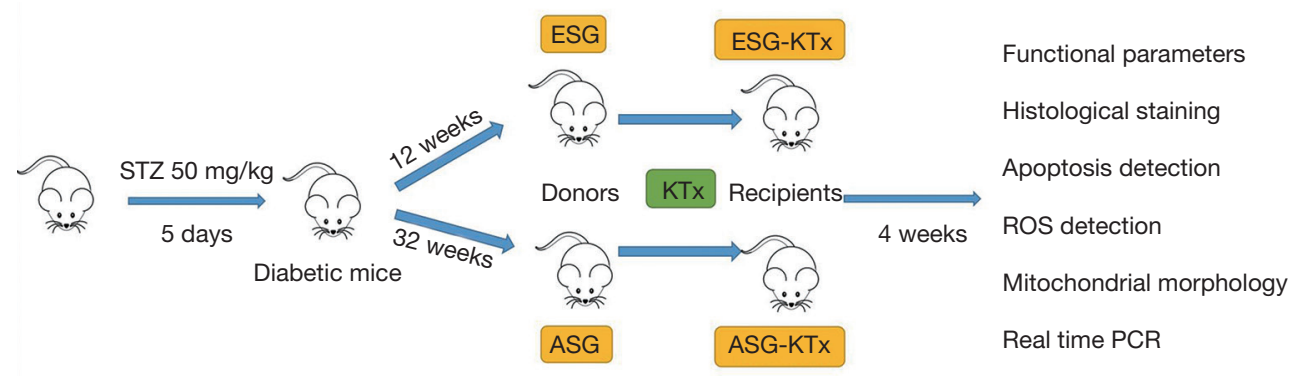

Figure 1 Animal experiment workflow. STZ, streptozotocin; ASG, advanced-stage group; ESG, early-stage; KTx, kidney transplantation; ROS, reactive oxygen species; PCR, polymerase chain reaction.

4\% paraformaldehyde, dehydrated in graded ethanol, paraffin embedded, and cut into 3-4- $\mu$ m-thick sections. These sections were stained with hematoxylin and eosin (H\&E), periodic acid Schiff (PAS), or Masson's trichrome. Digital images were analyzed and scored for glomerular mesangial expansion and mesangial matrix content using Image-Pro Plus 6.0 software (Media Cybernetics, Bethesda, MD, USA). Areas with positive PAS and aniline blue (indicating collagen fiber) staining were analyzed upon selection of an appropriate threshold. Values are reported as the ratio of positively stained glomerulus area to the total glomerular area.

\section{Detection of apoptosis and ROS}

Apoptosis was detected in formalin-fixed tissue sections with a TUNEL staining kit (In Situ Apoptosis Detection Kit; Abcam, Cambridge, MA, USA) according to the manufacturer's instructions. For ROS detection, unfixed frozen sections were incubated with the oxidative fluorescent dye dihydroethidium (DHE, $2 \mathrm{mmol} / \mathrm{L}$ ). The intensity of fluorescence was detected at $585 \mathrm{~nm}$ and quantified in the tissue sections under a confocal fluorescence microscope (DM 2500, Leica, Solms, Germany). The 3,3'-diaminobenzidine (DAB) control is shown in Figure S1.

\section{Electron microscopy}

Samples were fixed in a mixture of $4 \%$ paraformaldehyde and $0.5 \%$ glutaraldehyde in PBS, $\mathrm{pH}$ 7.4. The tissues were post-fixed for 2 hours in the same buffer containing $1 \% \mathrm{OsO} 4$. The tissue pieces were subsequently washed, dehydrated in graded acetone, and embedded in EponAraldite. Sections were prepared on an ultra-microtome, stained with $1 \%$ toluidine blue borax solution, mounted on copper grids, and double-stained with uranyl acetate. The mitochondrial morphological changes were observed under electron microscopy (Techai T10, Philips, Amsterdam, The Netherlands). The location in the kidney tissue was verified by an electron microscopy technician.

\section{Real-time quantitative reverse transcription polymerase chain reaction ( $q R T-P C R)$}

RNA was isolated from renal tissue using Trizol (Invitrogen, Carlsbad, CA, USA) and reverse transcribed using the RevertAid First Strand cDNA Synthesis Kit (Thermo Fisher Scientific, Waltham, MA, USA). The qRT-PCR analysis was performed using AceQ Universal SYBR qPCR Master Mix (Vazyme, Nanjing, China), gene-specific primers, and the C1000 Touch ${ }^{\mathrm{TM}}$ thermal cycler (Bio-Rad, Hercules, CA, USA). The data were normalized to GAPDH levels within each sample and analyzed using the $\Delta \Delta \mathrm{Ct}$ method. Primer sequences are listed in Table 1.

\section{Statistical analysis}

Results were expressed as means \pm SEM. One-way ANOVA followed by Scheffé's test or the Games-Howell test was used for comparison between groups with SPSS 22.0 for Windows; $\mathrm{P}<0.05$ was considered statistically significant.

\section{Results}

\section{Diabetic induction in mice}

Functional biochemical parameters before STZ injection (control) and 1 day before KTx (ESG and ASG) are shown in Figure 2. The levels of glycemia, serum Cre, BUN, and albuminuria of both ESG and ASG were significantly 
Table 1 Primers for quantitative real-time PCR

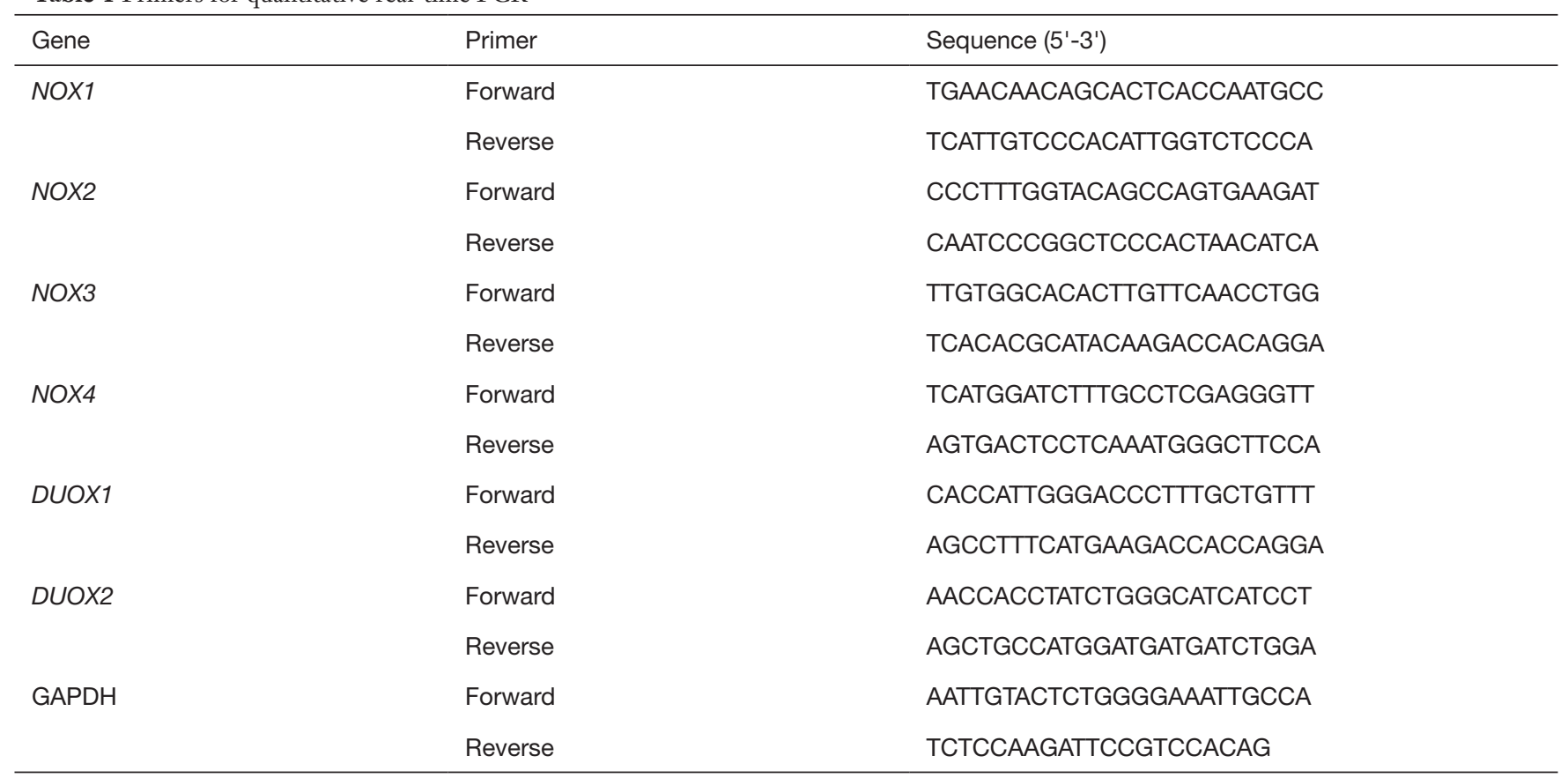

PCR, polymerase chain reaction.

increased after DM induction by STZ injection compared with those of the control group (Figure $2 A, B, C, D, E$ ). Although Cre and BUN were not significantly different between ESG and ASG, the albuminuria level was significantly higher $(\mathrm{P}=0.017)$, and body weight was further decreased in ASG compared with ESG $(\mathrm{P}=0.011)$. These data suggested that DM was induced in ESG and ASG.

Diabetic kidney features also became evident when pathological glomerular changes occurred, demonstrating more advanced DN in ASG than in ESG (Figure 3). The mesangial matrix and basement membrane areas were increased in both ESG and ASG compared with the control, with ASG showing more advanced changes, such as broader mesangial matrix expansion and a larger mesangial area (Figure 3G,I,K). In addition, nodular mesangial sclerosis was observed in ASG, but not in ESG (Figure 3M,O,Q).

\section{Functional parameters after KTx}

Functional biochemical parameters after KTx are shown in Figure 4. Levels of Cre and BUN in ESG and ASG were reduced after KTx compared with those in pre-KTx groups. We also noticed that albuminuria was significantly higher in ASG than in ESG $(\mathrm{P}<0.01)$.

\section{Reversal of diabetic changes in ESG kidneys after KTx}

To test the hypothesis that diabetic changes in the kidney can be reversed after transplant to a healthy recipient, we examined the glomerular matrix changes. For ESG kidneys, we found that diffused mesangial expansion and the thickened basement membrane were significantly attenuated at 4 weeks after $\mathrm{KTx}(\mathrm{P}<0.01)$, and these improvements were not observed in ASG kidneys $(\mathrm{P}=0.70)$ (Figure $3 G, H, I, \mathcal{F}, K, L, S)$. We also found that nodular mesangial sclerosis in ASG kidneys showed no significant changes after $\mathrm{KTx}(\mathrm{P}<0.01)$ (Figure $3 M, N, O, P, Q, R, T)$. These findings suggested that reversal of DN after transplant to a healthy individual was possible only in ESG.

\section{Effect of KTx on mitochondrial function}

Mitochondrial dysfunction has been proven to play an essential role in the development of DN (19). We evaluated the mitochondrial morphology of the kidneys in ESG and ASG before and after transplantation (Figure $5 A, B, C, D, E, F$ ). The normal mitochondria were claviform (Figure $5 A, B$ ). The mitochondria were bigger in ESG and ASG than in the control. We observed varying degrees of mitochondrial swelling in both diabetic groups before transplantation 
A

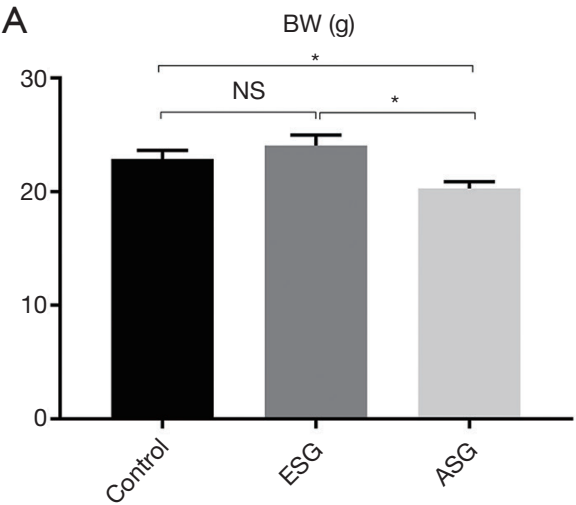

C

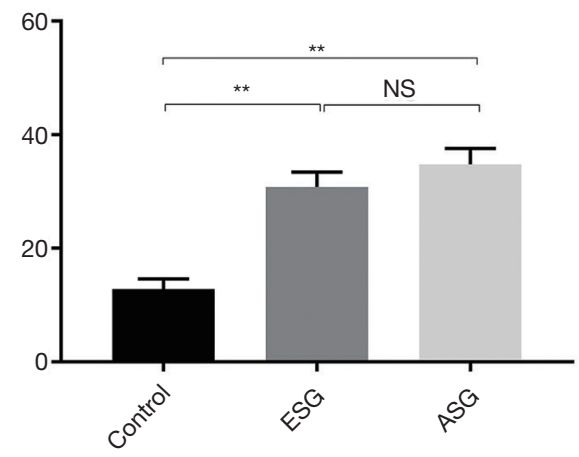

$E$

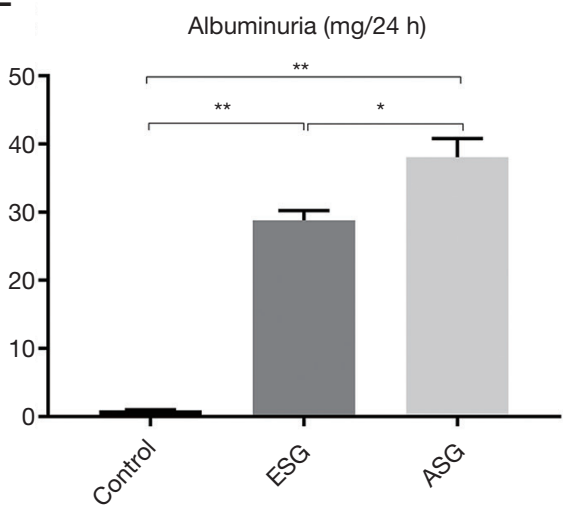

B

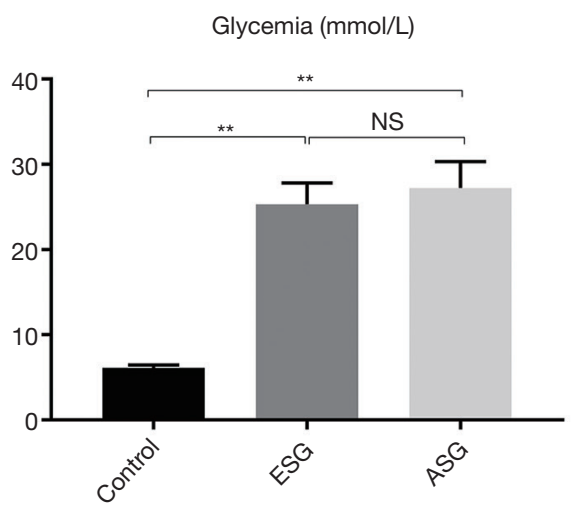

D

$\operatorname{BUN}(\mathrm{mg} / \mathrm{dL})$

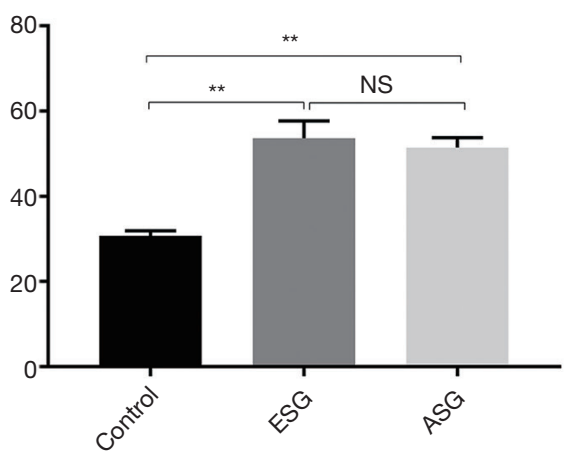

Figure 2 Metabolic data and biochemical parameters of the control, ESG, and ASG. (A) BW, (B) glycemia, (C) Cre, (D) BUN, (E) albuminuria. *, $\mathrm{P}<0.05$; ** $\mathrm{P}<0.01$. $\mathrm{n}=5$ mice per group. ESG, early-stage group; ASG, advanced-stage group; BW, body weight; Cre, creatinine; BUN, blood urea nitrogen.

(Figure 5C,E). Besides, the extreme mitochondrial swelling in ASG resulted in an oval shape. Furthermore, a small number of mitochondrial vacuoles and ruptured mitochondria were also found in ASG kidneys. After KTx, no swelling of mitochondria was observed in ESG, yet mitochondrial injury was not reduced in ASG kidneys
(Figure 5C,D,E,F). These results revealed that the reversal of $\mathrm{DN}$ is related to mitochondrial function.

To further confirm the difference in mitochondrial function between ESG and ASG, we examined ROS formation by detection of superoxide anion production through DHE staining and apoptosis by TUNEL staining 


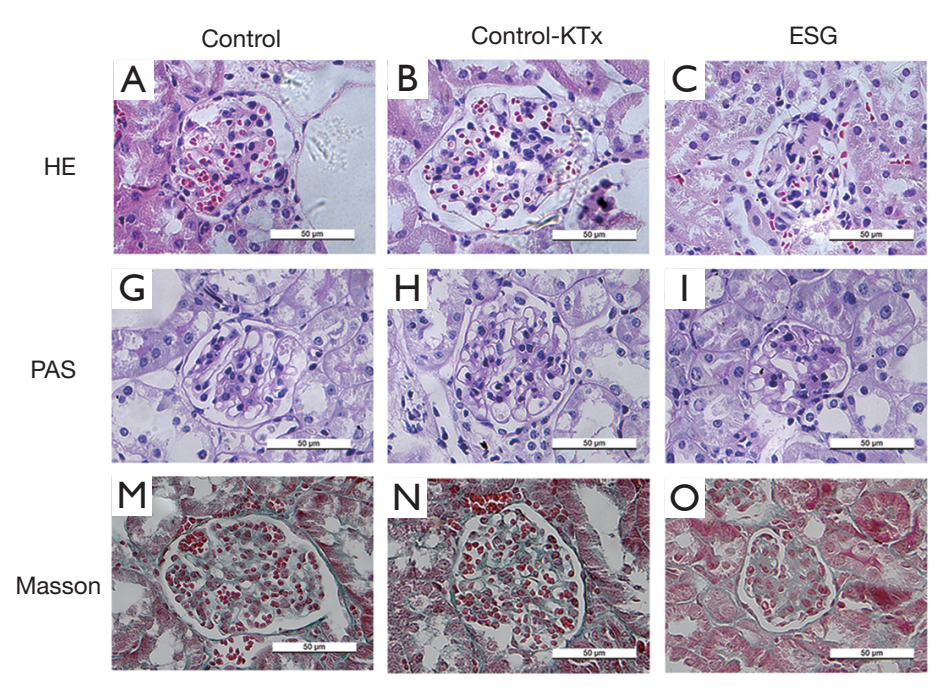

$S$

The relative area of mesangium matrix and basement membrane

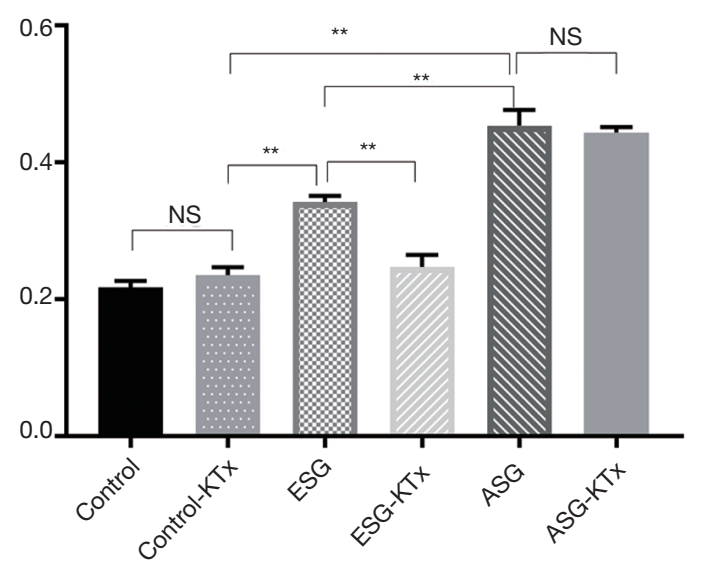

$T$
ASG-KTX
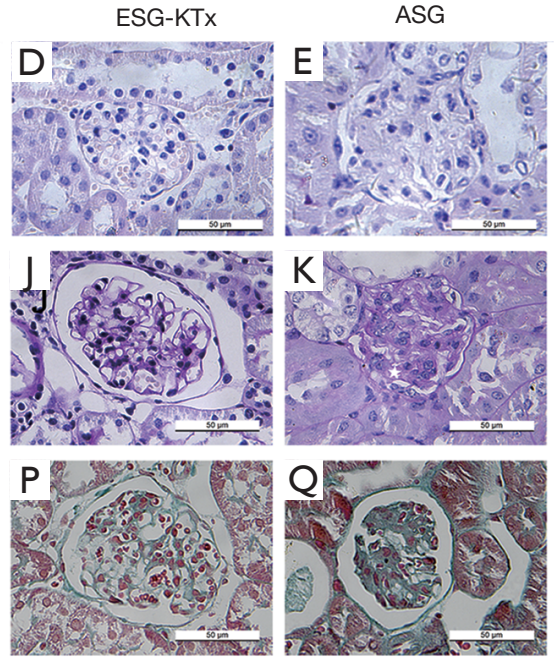
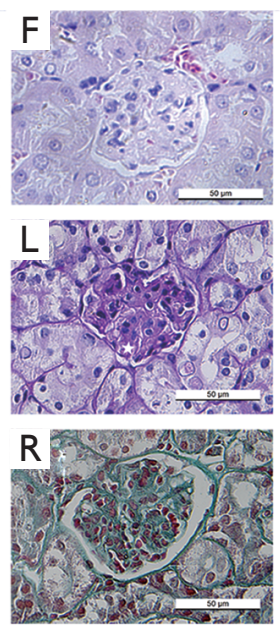

The relative area of fibrosis

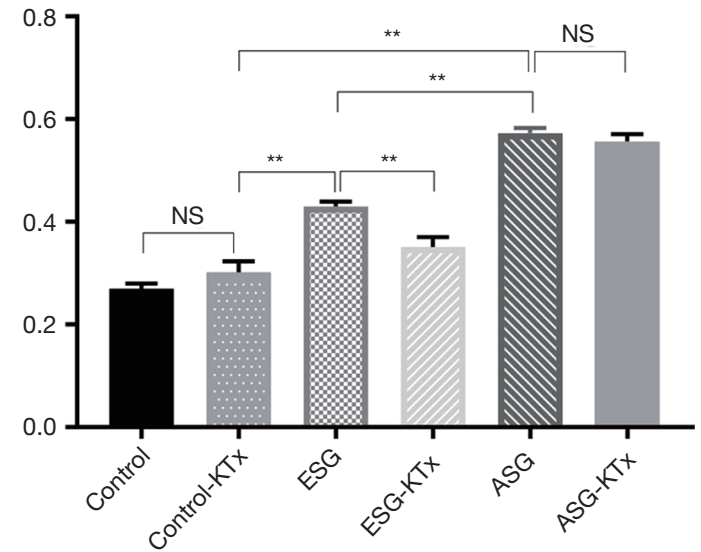

Figure 3 Glomerular pathological changes in ESG and ASG before and after KTx and control mice. Representative images of renal morphological changes: tissue sections stained by hematoxylin and eosin (H\&E) (A,B,C,D,E,F), glycogen accumulation stained by periodic acid Schiff (PAS) (G,H,I,J,K,L), and interstitial fibrosis stained by Masson's trichrome (M,N,O,P,Q,R). Stars show nodular mesangial sclerosis (K,L). Magnification: 400x. Relative area of mesangial matrix and basement membrane (S) and renal interstitial fibrosis (T) at different times was quantified and is presented in bar graphs. ${ }^{* *}, \mathrm{P}<0.01$. $\mathrm{n}=5$ mice per group. ESG, early-stage group; ASG, advanced-stage group; KTx, kidney transplantation.

(Figure $5 G, H, I, \mathcal{F}, K, L, M, N, O, P, Q, R)$. We observed a significant increase in ROS production in both ESG $(\mathrm{P}<0.01)$ and ASG $(\mathrm{P}<0.01)$ kidneys (Figure $5 G, I, K)$. Besides, ASG kidneys showed a higher level of ROS production than ESG kidneys $(\mathrm{P}<0.05)$. In line with the morphological changes, the ROS production in the kidneys of ESG was attenuated after $\mathrm{KTx}(\mathrm{P}<0.05)$, but no remarkable changes in ROS were observed in the kidneys of ASG $(\mathrm{P}=0.6)$ (Figure $5 G, H, I, \mathcal{F}, K, L, S)$. Analysis of TUNEL staining in the renal tissues of mice showed significant suppression of apoptosis in ESG $(\mathrm{P}<0.01)$ but not ASG after KTx $(\mathrm{P}=0.77)$ (Figure $5 M, N, O, P, Q, R, T)$. Positive cells were observed in glomeruli of both ESG and ASG, but ASG showed considerably more positive cells. KTx also suppressed 
A

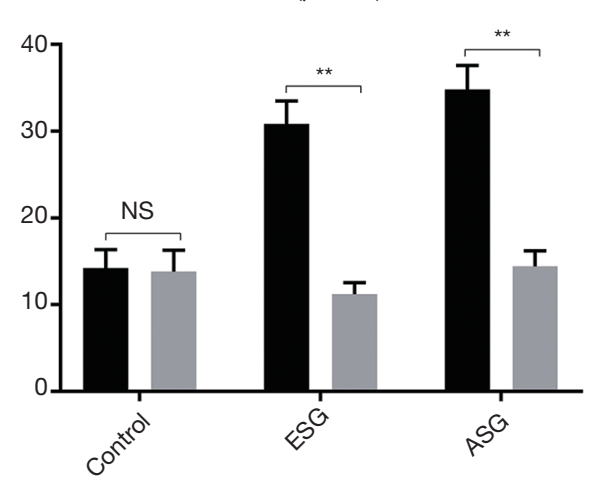

C

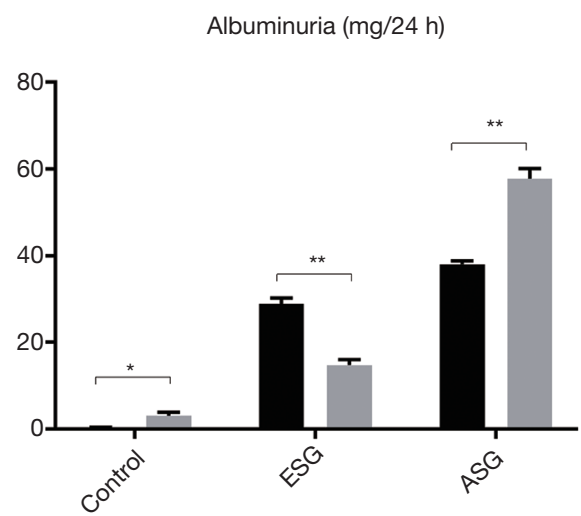

B

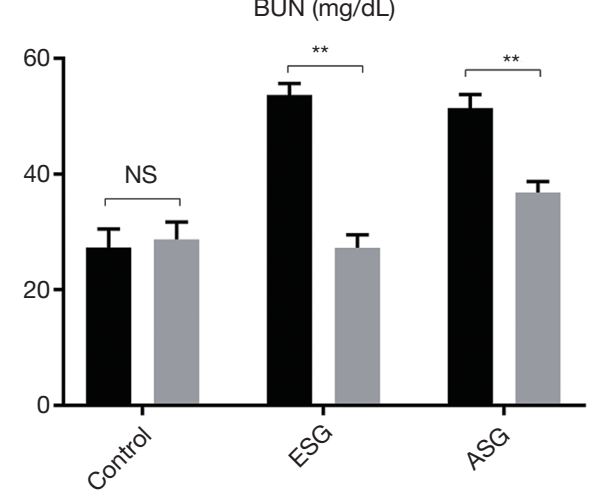

Figure 4 Metabolic data and biochemical parameters of ESG and ASG before and after KTx. (A) Cre, (B) BUN, (C) albuminuria. Control: the kidneys of 8-week-old mice were transplanted into age-matched mice. On the left, Cre, BUN and albuminuria in pre-KTx cohort; on the right, the post-KTx cohort. *, $\mathrm{P}<0.05$; **, $\mathrm{P}<0.01$. n=5 mice per group. ESG, early-stage group; ASG, advanced-stage group; KTx, kidney transplantation.

apoptosis in ESG but not in ASG.

\section{Effect of KTx on NOXs}

NOXs catalyze the transfer of electrons from NADPH to molecular oxygen to produce superoxide and/or hydrogen peroxide, two major ROS (20). Therefore, we examined the transcriptional levels of the NOX family (Figure 6A,B,C,D,E,F). We found that the expression of NOX1, NOX2, NOX3, and NOX4 was significantly increased in ESG and ASG when compared with the control. The levels of NOX1 $(\mathrm{P}<0.05)$, NOX3 $(\mathrm{P}<0.05)$, and NOX4 $(\mathrm{P}<0.01)$ in ASG were significantly higher than in ESG, whereas there was no difference in NOX2 expression between ESG and ASG $(\mathrm{P}=0.10)$. In addition, only NOX1 $(\mathrm{P}<0.01)$, NOX2 $(\mathrm{P}<0.001)$, and NOX4 $(\mathrm{P}<0.05)$ expression was reduced in ESG after KTx. Moreover, DUOX1 expression was only increased in ASG $(\mathrm{P}<0.05)$ and not inhibited after KTx $(\mathrm{P}=0.44)$. DUOX2 expression was higher in ASG than ESG $(\mathrm{P}<0.05)$ but was not changed after KTx in both ESG and ASG. Inhibition of NOX1 and NOX4 results in reduced albuminuria, glomerular hypertrophy, and mesangial matrix accumulation (21), which was in line with our findings.

\section{Discussion}

In this study, we evaluated the reversibility of diabetic changes in kidney grafts from diabetic mice after KTx. Mildly diffused mesangial expansion and the thickened basement membrane were attenuated after KTx, resulting in improved function. We further found that only early DN was reversible after KTx. In addition, we confirmed that advanced lesions, such as nodular mesangial sclerosis, 
Control

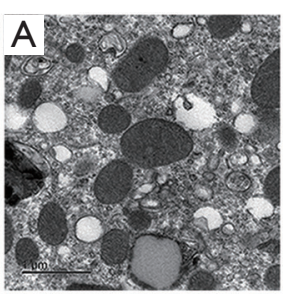

G
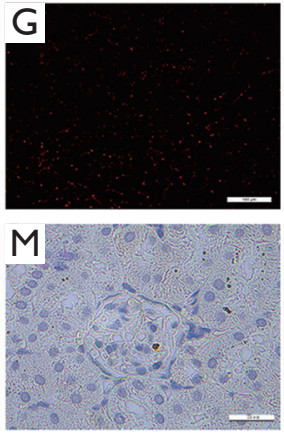

S DHE fluorescence (a u.)
ESG
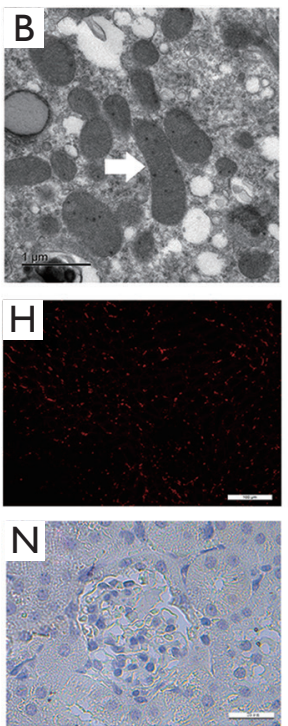
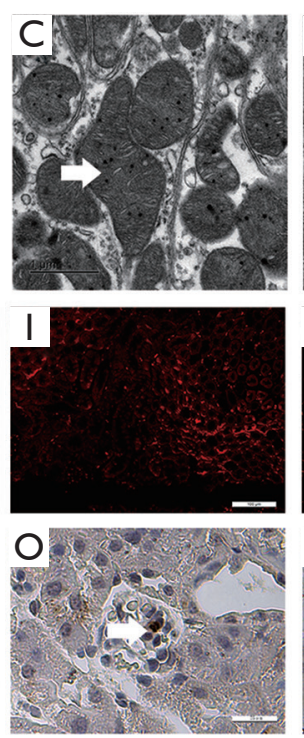

\section{.}

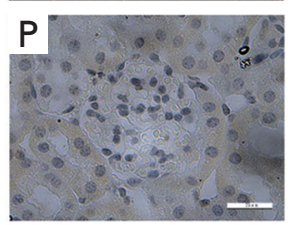

$\mathrm{T}$

ESG-KTx
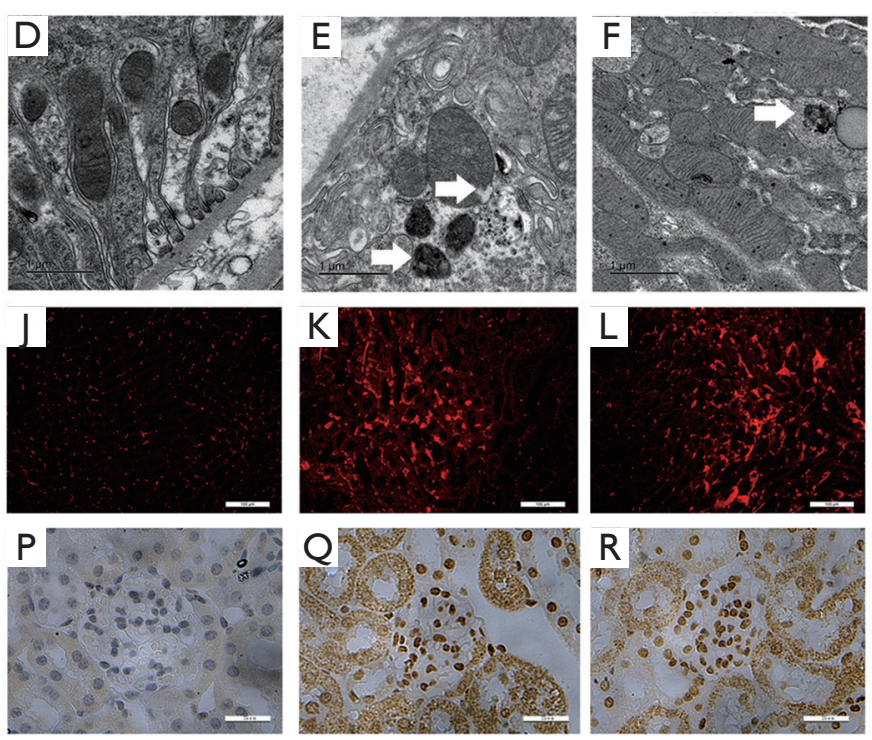

Glomerulus (cells/gcs)
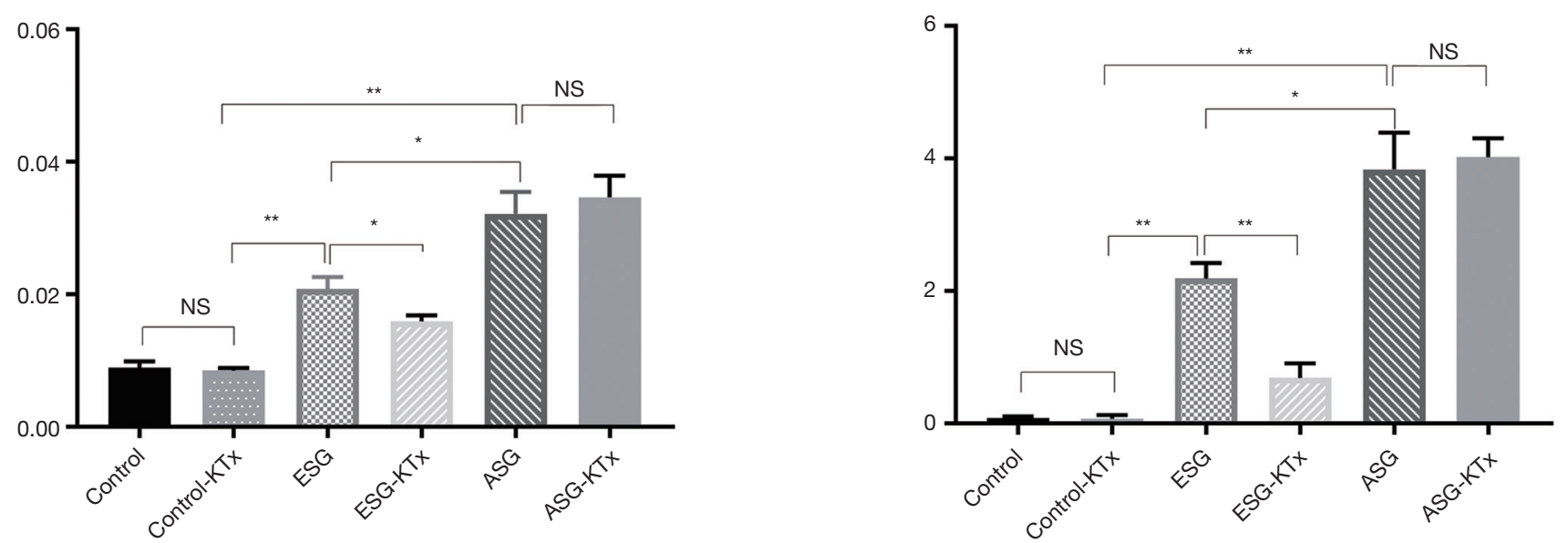

Figure 5 The mitochondrial morphology mitochondrial morphology of the kidneys in ESG and ASG before and after transplantation was evaluated through electron microscopy. (A,B,C,D,E,F) Representative mitochondria of kidney tissues from ESG and ASG before and after KTx. Arrows show a normal mitochondrion (B), an extremely large mitochondrion (C), a mitochondrial vacuole (E), and a ruptured mitochondrion (F). Magnification: 10,000x. (G,H,I,J,K,L) Detection of superoxide formation in kidney sections by DHE staining. The arrow shows a positively stained cell. Magnification: 100×. (M,N,O,P,Q,R) Apoptosis detection by TUNEL assay. Magnification: 400×. The relative intensity of DHE fluorescence $(\mathrm{S})$ and apoptotic cells in kidney tissues $(\mathrm{T})$ were quantified and are presented in bar graphs. *, $\mathrm{P}<0.05$; ** $\mathrm{P}<0.01$. $\mathrm{n}=5$ mice per group. ESG, early-stage group; ASG, advanced-stage group; KTx, kidney transplantation. 
A

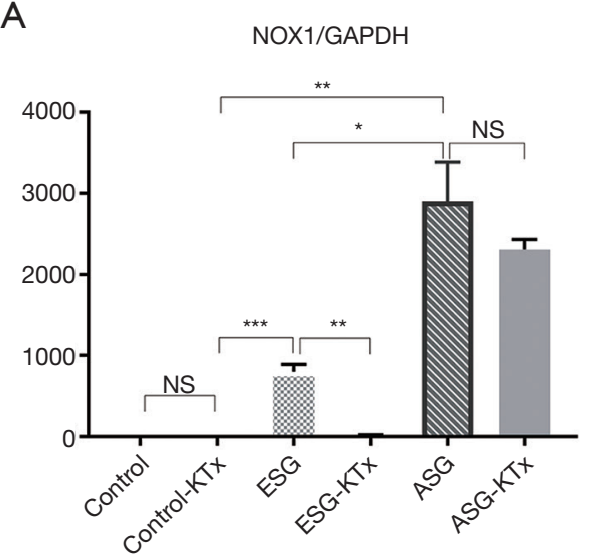

C

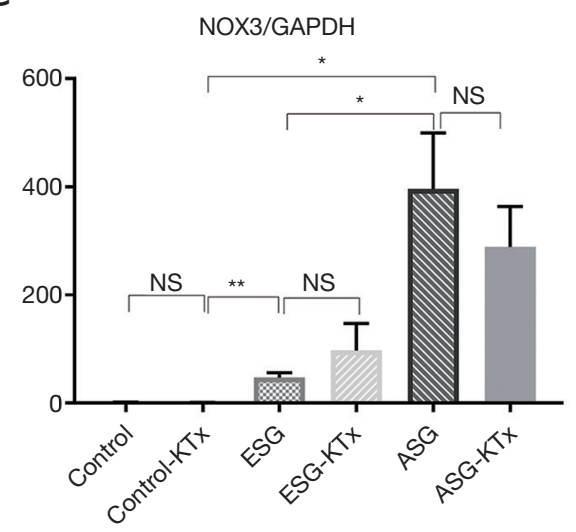

$\mathrm{E}$

DUOX1/GAPDH

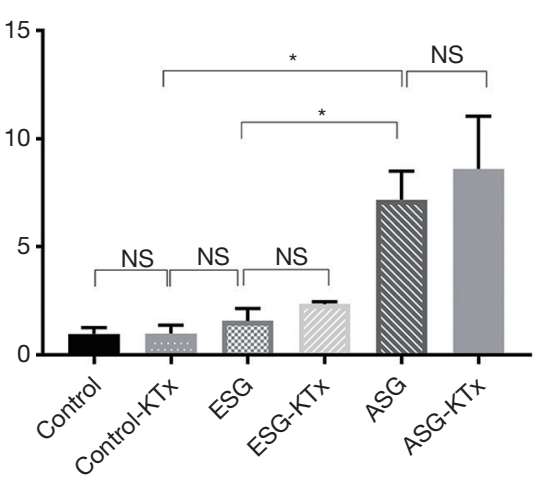

B

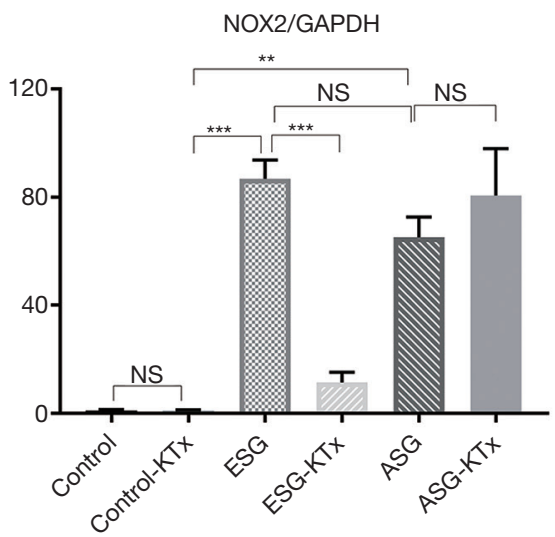

D

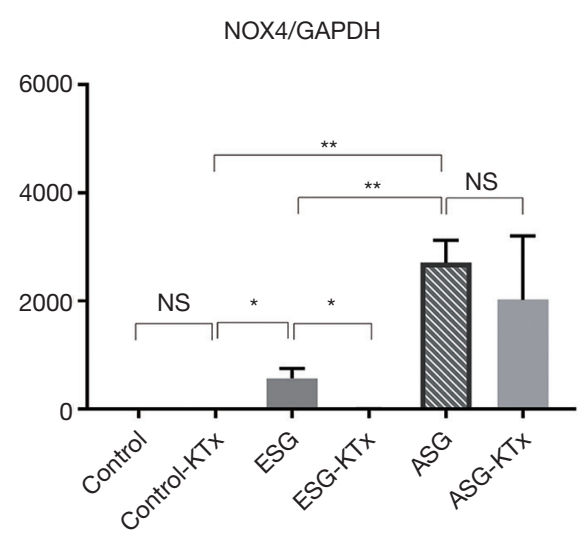

$\mathrm{F}$

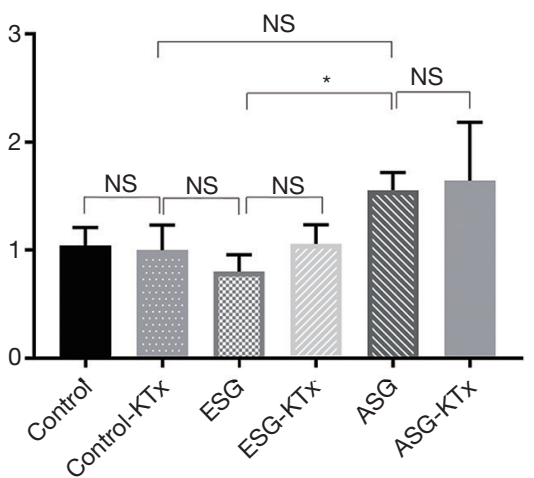

Figure 6 Relative transcriptional levels of NOX family. (A) NOX1, (B) NOX2, (C) NOX3, (D) NOX4, (E) DUOX1, (F) DUOX2. *, $\mathrm{P}<0.05$; ** $\mathrm{P}<0.01$, *** $\mathrm{P}<0.001$. $\mathrm{n}=3$ mice per group. NOX, NADPH oxidase; $\mathrm{DUOX}$, dual oxidase. 
were irreversible in mice. These results may be related to excessive oxidative stress damage in the tissues, i.e., mitochondrial function, which was induced by overexpression of NOXs.

Lee et al. have reported a similar phenomenon (9), observing that glomerular lesions were reversed after KTx. We further considered DN patients of stage 1 or $2 \mathrm{a}$ as "early-stage," whereas stage 3 or 4 was considered as "advanced-stage." However, despite extensive similarities, there is no exact correspondence between mice and human patients. Renal failure is not as prominent in mice with $\mathrm{DN}$ as it is in humans (17). In this study, increased plasma Cre and BUN were observed, but there was no difference between ESG and ASG. They were distinguished mainly by pathologic changes, mild basement membrane thickening, and mesangial expansion in ESG (the relative areas of mesangial matrix and basement membrane in ESG were below 0.35 ) and a mass of mesangial expansion and mesangial sclerosis in ASG (the relative areas of mesangial matrix and basement membrane in ESG were beyond 0.4). Besides, bodyweight decline was another feature of ASG, which only occurs in mice whose disease course is more than 30 weeks. Moreover, inhibition of ROS and NOXs was confirmed to be relative to the reversibility.

The main characteristics of $\mathrm{DN}$ include the accumulation of extracellular matrix (ECM) in the mesangium, glomerular and tubular basement membranes, and interstitium under hyperglycemic conditions (22). Conway et al. reported that strict blood glycemic control attenuated ECM in DN under experimental conditions (23). Thus, normoglycemia after KTx appears to improve early diabetic changes, which was supported by our findings. Nonetheless, whether intensive glycemic control can improve the prognosis of diabetic patients is under debate. However, glycemic control increases the risk of hypoglycemia (24). Reversal of DN after KTx is not only dependent on low glucose levels; it was also found that excessive ROS production plays a vital role in the occurrence and development of DN and thus must be attenuated to reverse DN (19). In the hyperglycemic environment, dysfunction of the mitochondria leads to overwhelming production of ROS, and the antioxidant defense systems are quickly exhausted, favoring ROS accumulation outside of mitochondria (15). The target cells, including glomerular mesangial cells, capillary endothelial cells of the retina, and neuronal cells, are unable to adequately regulate the concentrations of intracellular glucose in the diabetic environment.

Similarly to direct injury, overwhelming oxidative stress in the state of hyperglycemia damages the mitochondrial genetic material (mtDNA) and nuclear DNA (nDNA) (25) and finally induces apoptosis in various types of cells (26). Apoptosis has been observed in tubular epithelial cells, endothelial cells, and interstitial cells in renal biopsies from patients with DN (27). There are two major pathways of apoptosis: the extrinsic pathway and the intrinsic pathway. The extrinsic pathway involves the interaction of death-inducing ligands and their corresponding death receptors on the plasma membrane (28). In the intrinsic pathway, the mitochondrion is the central regulator (29). When mitochondria are injured, cytochrome $\mathrm{c}$ is released into the cytoplasm to induce the extrinsic pathway (30), whereas damaged mitochondria directly induce the intrinsic pathway. In this study, after transplantation into a standard mouse, the ESG kidneys were removed from the hyperglycemic environment and exposed to less oxidative stress, resulting in recovery of kidney function. Thus, KTx provides a normal internal milieu and prevents the sustained injuries caused by ROS and apoptosis. For advanced DN lesions, overwhelming oxidative stress caused considerably more severe and sustained damage to the kidney; therefore, the changes appeared to be irreversible, even after adverse factors were removed.

NOXs are strong contributors to ROS formation in the diabetic kidney (15). NOX2 in particular plays a vital role in neutrophil function (31). NOX4 is the predominant NOX isoform expressed in the kidney (14), and increased expression thereof was observed in various kidney cells cultured in high-glucose media and kidney tissue from different rodent models of diabetes (32). Inhibition of NOX1 and NOX4 has been shown to reduce ROS production in $\mathrm{db} / \mathrm{db}$ mice (33), and global deletion of NOX4 attenuated albuminuria and glomerular injury in STZ-treated ApoE-/- mice (34). Furthermore, inhibition of NOXs results in reduced ROS formation and prevents apoptosis (16). In this study, most NOXs were overexpressed in diabetic mice, and levels of NOX1 and NOX4 were significantly higher in ASG than in ESG. In the ESG, the average blood glucose after KTx blocked the hyperglycemia-NOXs-ROS axis. Not only the direct effects of ROS but also molecules activated by NOXs, such as a-SMA, collagen IV, and fibronectin, were inhibited (21). Furthermore, systemic inflammation caused by hyperglycemia and NOXs was depressed (35). Lesions such as mild basement membrane thickening and mesangial expansion were finally reversed.

We acknowledge that our study has several limitations. As one kidney of each recipient was maintained, it was 
not clear whether the renal donor graft was functional. However, the existence of albuminuria indicated that the graft participated in filtration. Otherwise, the increased albuminuria in ASG after KTx may be the result of further damage following KTx. Although we found that early DN was reversible, whereas advanced DN was irreversible, we did not take any further steps to develop criteria of useful kidneys.

\section{Conclusions}

$\mathrm{DN}$ in kidneys from early-stage diabetic mice is reversible after $\mathrm{KTx}$, whereas that from advanced-stage diabetic mice is not. This phenomenon is related to improvement in mitochondrial function and suppression of NOXs. This study suggests that only early-stage DN patients should be considered as kidney donors, and advanced-stage DN patients should be excluded. Nonetheless, further studies should be performed to establish the criteria of useful kidneys and to further elucidate the underlying mechanism of DN reversal.

\section{Acknowledgments}

We are grateful to all staff of the Kidney Disease Center of the First Affiliated Hospital of Zhejiang University for their sincere supports.

Funding: This work was supported by grants from the National Natural Science Foundation of China (No. 81470938, 81770697, 81770752, 81770750).

\section{Footnote}

Conflicts of Interest: The authors have no conflicts of interest to declare.

Ethical Statement: The authors are accountable for all aspects of the work in ensuring that questions related to the accuracy or integrity of any part of the work are appropriately investigated and resolved. The animal experiments were approved by the Institution Ethics Committee for Investigation with Animals (Zhejiang University) (No. 2019630).

\section{References}

1. Zhang L, Long J, Jiang W, et al. Trends in Chronic Kidney Disease in China. N Engl J Med 2016;375:905-6.
2. Nathan DM, Genuth S, Lachin J, et al. The effect of intensive treatment of diabetes on the development and progression of long-term complications in insulin-dependent diabetes mellitus. $\mathrm{N}$ Engl J Med 1993;329:977-86.

3. Intensive blood-glucose control with sulphonylureas or insulin compared with conventional treatment and risk of complications in patients with type 2 diabetes (UKPDS 33). UK Prospective Diabetes Study (UKPDS) Group. Lancet 1998;352:837-53.

4. Steffes M, Chavers B, Molitch M. Sustained effect of intensive treatment of type 1 diabetes mellitus on development and progression of diabetic nephropathy: the Epidemiology of Diabetes Interventions and Complications (EDIC) study. JAMA 2003;290:2159-67.

5. Fioretto P, Mauer M. Effects of pancreas transplantation on the prevention and reversal of diabetic nephropathy. Contrib Nephrol 2011;170:237-46.

6. Fioretto P, Mauer M. Reversal of diabetic nephropathy: lessons from pancreas transplantation. J Nephrol 2012;25:13-8.

7. Abouna GM, Al-Adnani MS, Kremer GD, et al. Reversal of diabetic nephropathy in human cadaveric kidneys after transplantation into non-diabetic recipients. Lancet 1983;2:1274-6.

8. Harada S, Ushigome H, Nishimura A, et al. Histological reversibility of diabetic nephropathy after kidney transplantation from diabetic donor to non-diabetic recipient. Nephrology 2015;20:40-4.

9. Lee CS, Mauer SM, Brown DM, et al. Renal transplantation in diabetes mellitus in rats. J Exp Med 1974;139:793-800.

10. Pagliarini DJ, Calvo SE, Chang B, et al. A mitochondrial protein compendium elucidates complex I disease biology. Cell 2008;134:112-23.

11. Reidy K, Kang HM, Hostetter T, et al. Molecular mechanisms of diabetic kidney disease. J Clin Invest 2014;124:2333-40.

12. Lee AY, Chung SS. Contributions of polyol pathway to oxidative stress in diabetic cataract. FASEB J 1999;13:23-30.

13. Bedard K, Krause KH. The NOX family of ROSgenerating NADPH oxidases: physiology and pathophysiology. Physiol Rev 2007;87:245-313.

14. Shiose A, Kuroda J, Tsuruya K, et al. A novel superoxideproducing $\mathrm{NAD}(\mathrm{P}) \mathrm{H}$ oxidase in kidney. J Biol Chem 2001;276:1417-23.

15. Forbes JM, Coughlan MT, Cooper ME. Oxidative stress 
as a major culprit in kidney disease in diabetes. Diabetes 2008;57:1446-54.

16. Susztak K, Raff AC, Schiffer M, et al. Glucose-induced reactive oxygen species cause apoptosis of podocytes and podocyte depletion at the onset of diabetic nephropathy. Diabetes 2006;55:225-33.

17. Breyer MD, Bottinger E, Brosius FC 3rd, et al. Mouse models of diabetic nephropathy. J Am Soc Nephrol 2005;16:27-45.

18. Tse GH, Hesketh EE, Clay M, et al. Mouse kidney transplantation: models of allograft rejection. J Vis Exp 2014:e52163.

19. Qi H, Casalena G, Shi S, et al. Glomerular Endothelial Mitochondrial Dysfunction Is Essential and Characteristic of Diabetic Kidney Disease Susceptibility. Diabetes 2017;66:763-78.

20. Lambeth JD, Neish AS. Nox enzymes and new thinking on reactive oxygen: a double-edged sword revisited. Annu Rev Pathol 2014;9:119-45.

21. You YH, Quach T, Saito R, et al. Metabolomics Reveals a Key Role for Fumarate in Mediating the Effects of NADPH Oxidase 4 in Diabetic Kidney Disease. J Am Soc Nephrol 2016;27:466-81.

22. Fioretto P, Mauer M. Histopathology of diabetic nephropathy. Semin Nephrol 2007;27:195-207.

23. Conway BR, Betz B, Sheldrake TA, et al. Tight blood glycaemic and blood pressure control in experimental diabetic nephropathy reduces extracellular matrix production without regression of fibrosis. Nephrology (Carlton) 2014;19:802-13.

24. Hemmingsen B, Lund SS, Gluud C, et al. Intensive glycaemic control for patients with type 2 diabetes: systematic review with meta-analysis and trial sequential analysis of randomised clinical trials. BMJ 2011;343:d6898.
25. Fernyhough P, Huang TJ, Verkhratsky A. Mechanism of mitochondrial dysfunction in diabetic sensory neuropathy. J Peripher Nerv Syst 2003;8:227-35.

26. Wagener FA, Dekker D, Berden JH, et al. The role of reactive oxygen species in apoptosis of the diabetic kidney. Apoptosis 2009;14:1451-8.

27. Allen DA, Harwood S, Varagunam M, et al. High glucoseinduced oxidative stress causes apoptosis in proximal tubular epithelial cells and is mediated by multiple caspases. FASEB J 2003;17:908-10.

28. Ashkenazi A, Dixit VM. Death receptors: signaling and modulation. Science 1998;281:1305-8.

29. Jin Z, El-Deiry WS. Overview of cell death signaling pathways. Cancer Biol Ther 2005;4:139-63.

30. Li H, Zhu H, Xu CJ, et al. Cleavage of BID by caspase 8 mediates the mitochondrial damage in the Fas pathway of apoptosis. Cell 1998;94:491-501.

31. Winterbourn CC, Kettle AJ, Hampton MB. Reactive Oxygen Species and Neutrophil Function. Annu Rev Biochem 2016;85:765-92.

32. Rhee EP. NADPH Oxidase 4 at the Nexus of Diabetes, Reactive Oxygen Species, and Renal Metabolism. J Am Soc Nephrol 2016;27:337-9.

33. Sedeek M, Gutsol A, Montezano AC, et al. Renoprotective effects of a novel Nox1/4 inhibitor in a mouse model of Type 2 diabetes. Clin Sci (Lond) 2013;124:191-202.

34. Jha JC, Gray SP, Barit D, et al. Genetic targeting or pharmacologic inhibition of NADPH oxidase nox4 provides renoprotection in long-term diabetic nephropathy. J Am Soc Nephrol 2014;25:1237-54.

35. Edlund J, Fasching A, Liss P, et al. The roles of NADPHoxidase and nNOS for the increased oxidative stress and the oxygen consumption in the diabetic kidney. Diabetes Metab Res Rev 2010;26:349-56.
Cite this article as: $\mathrm{Wu} \mathrm{W}$, Jin $\mathrm{Y}$, Teng L, Shao X, Wang Y, Feng S, Wang C, Jiang H, Wu J. Mitochondria-related reversal of early-stage diabetic nephropathy in donor kidney after transplantation in mice. Ann Transl Med 2019;7(24):801. doi: 10.21037/atm.2019.12.55 

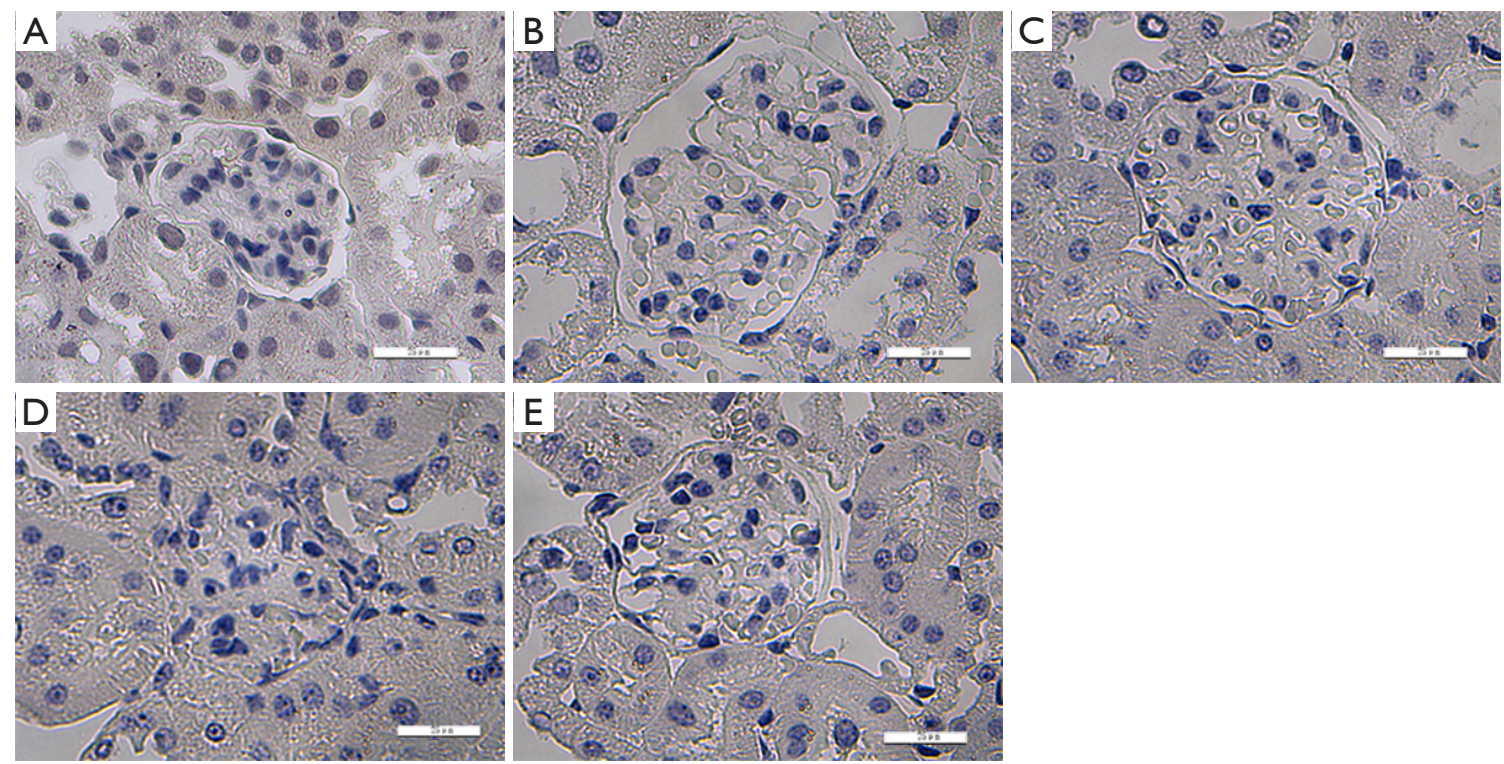

Figure S1 DAB controls for control (A), ESG (B), ESG-KTx (C), ASG (D) and ASG-KTx (E).These sections were only stained by DAB and hematoxylin. Magnification: 400x. ESG, early-stage group; ASG, advanced-stage group; KTx, kidney transplantation; DAB, 3,3'-diaminobenzidine. 